\title{
Research on Digital Cultural Creation Design of The Palace Museum from the Perspective of Symbol consumption -- A Case study of Twelve Beauties of Prince Yong
}

\author{
Gang li $^{1 a^{*}}$,Jiaqi Xuan ${ }^{2 b}$, Shaoyang $\operatorname{Ren}^{3 \mathrm{c}}$ \\ ${ }^{1}$ Collage of art Chongqing university, Chongqing, China \\ ${ }^{2}$ Collage of art Chongqing university, Chongqing, China \\ ${ }^{3}$ Collage of art Chongqing university, Chongqing, China
}

\begin{abstract}
With the development of digital technology, mobile terminals have become a new medium for museum cultural and creative products to spread the connotation of art and culture. The innovation of digital technology has caused corresponding changes in the design of cultural and creative products, and various digital cultural and creative products have been created on its basis. The emergence of digital technologies such as mobile Internet, cloud computing, AI, VR, human-computer interaction, and 5G communication technology has brought broader development prospects for digital creative design. This article studies the concepts of cultural symbols and design methods of the digital cultural and creative products of The Palace Museum to provide new ideas for the design of digital cultural and creative products of the museum. We choose Twelve Beauties of Prince Yong as an example, the first digital cultural creation product of The Palace Museum.
\end{abstract}

\section{INTRODUCTION}

\subsection{Background overview}

Since the reform and opening up, the economy of Chinese market has developed rapidly with increasingly frequent economic and cultural exchanges with other countries. When people's physiological needs can be satisfied, they will have higher demands. They will pursue the added value and significance of commodities to meet their spiritual needs, and even become "consumption for consumption's sake". Their consumption behavior gradually changes from using value consumption to symbol value consumption. Especially in the era of new mobile media. As the country with the most smartphone users in the world, China is developing into the world's largest mobile Internet market. The main form of modern information generation and dissemination is to rely on digital media technology that is also one of the most advanced productive forces in the new century and plays a vital role in promoting the development of creative industries. The use of mobile phones and other mobile electronic devices has changed the reading habits of most modern people. Unlike traditional art appreciation or reading habits, modern people tend to read in fragments. People tend to have diverse and simple cultural and artistic experiences through new media. The reproducibility of images in the age of mechanical reproduction and the maturity of new media technology make the exhibition space of mobile application products a channel of information, in which people can flow for art appreciation and information feedback and break through the limitation of time and space [1]

\subsection{Summary of cultural and creative Industry of the Palace Museum.}

The Palace Museum, known as the Forbidden City, is the essence of ancient Chinese architecture. The Palace Museum has a complete collection of ancient and modern cultural relics of excellent quality and rich varieties. The total amount of the collection has reached 1863,404 pieces (sets), mainly including cultural relics of the Ming and Qing dynasties, ancient architecture and books. The Palace Museum is the quintessence of Chinese culture for thousands of years into the museum, is a hot IP. After early exploration and development, the Cultural and creative works of the Palace Museum began to receive increasing attention in 2011. By 2018, the Palace Museum had developed more than 11,900 products, with the sales volume of cultural and creative works reaching 1.5 billion yuan. In this era of "mixing" and "crossover", with the further deepening of "Internet +", museums are increasingly aware that a single original exhibition form can no longer meet the growing cultural consumption demand of the public. Therefore, it is necessary for museums to transmit the cultural connotation of cultural

\footnotetext{
a*704427388@qq.com

b392921656@qq.com

c1049434388@qq.com
} 
relics to the public in various ways. In May 2013, the first APP produced by the Palace Museum featuring the screen of Twelve Beauties of Prince Yong, was downloaded more than 200,000 times within two weeks after its launch. The Palace Museum has released 10 apps in succession. Twelve Beauties of Prince Yong, Auspicious Symbols in the Forbidden City, Emperor for a Day, Night Revels of Han Xizai, 365 Days of Masterpieces and The Qing Emperor's Wardrobe were all recommended by The Apple Store and became the best apps for audiences, which also made the Palace Museum become a cultural brand expected and followed by the public.

\subsection{Literature review of the Palace Museum}

At present, Chinese scholars pay more attention to cultural and creative products. Especially, with the success of cultural and creative products in the Palace Museum, more and more researchers pay attention to them. There are four perspectives on the study of cultural creation in the Palace Museum: One is the research on the development and design of cultural and creative products or cultural and creative industries of the Palace Museum; The second category is the marketing strategy and brand management of the Cultural and creative of the Palace Museum; The third category is the study on the artistic design and aesthetics of cultural and creative products of the Palace Museum; The fourth category is the research on the social effect and cultural communication of the cultural creation of the Palace Museum. As for the study of cultural creation in in the Palace Museum by using the theory of semiotics, it mainly analyzes and interprets the content of cultural creation in the Palace Museum from the perspectives of using semiotics to analyze the pattern or product design of cultural creation in the Palace Museum, semiotics content analysis and communication mode, etc. However, under the background of mobile new media technology, especially in the era of largescale symbol consumption, cultural and creative products have more cultural symbol connotation and marketing communication means than before.

\section{The Design Theory and TeChNiCAL FRAMEWORK OF DIGITAL CULTURAL Creation of the Palace Museum}

\subsection{The Symbol Consumption Theory in the design of digital cultural creative product of the Palace Museum}

French sociologist Roland Barthes made a detailed introduction to the signifier process of symbols in his book Principles of Semiotics. On the basis of Ye Erm Lev ideas of semiotics, he points out that two signifier systems of symbols. Direct signifier constitutes the first system, and intentional signifier constitutes the second system. He proposed that "a system with intentional reference is a system whose expression surface is composed of a system of intentional reference", and "in Semiotics with intentional reference, the signifier of the second system is composed of the marks in the first system." Barthes' definition of the two systems and their relationship enriches the connotation of the symbol itself and the extension of its symbolic meaning [2]. Therefore, In addition to its own use value, the Palace Museum digital cultural and creative products are also a kind of cultural symbol communication, an aesthetic emotional experience, the pursuit of the masses for things gradually transformed into the pursuit of symbol value beyond the objects themselves contained behind them, so as to seek self-identity, show consumption level and highlight social status through the meaning of symbols.

The Digital cultural creation of the Palace Museum decoded the cultural significance of cultural relics in its collection and reconstructed it with mobile digital media technology and modern reading habits that are easily accepted by the public. Based on the rich collection resources, the original cultural symbols of cultural relics are extracted and represented in the symbol space of digital products, and the display content is stratified by using interactive media to encode symbols in different forms such as images, videos and audio. In addition to reflecting functional association in general information display, digital cultural and creative products of the Palace Museum also need to extract cultural meaning and aesthetic emotion to interact with symbols applied in artistic content. The interactive products formed on this basis enable users to generate positive cognitive response, decode the meaning of symbols, generate expected interactive behavior, and constitute the corresponding symbol consumption system. At present, the value of digital cultural creations in the Palace Museum has changed from "the use of practical functions" to "the construction of symbol consumption and aesthetic experience emotions".

\subsection{Technology framework of digital cultural creative products design of the Palace Museum}

The application - the Palace Museum, is a new development of digital cultural creative products. Various museum apps conform to the new trend of the integration of culture and technology, providing a new platform for information integration and networking, information display and multimedia, real-time information update and interactive information experience [3]. At present, according to the development and use of the Palace Museum creative application, some applications are suitable for Android and iPhone, such as Emperor for a Day, 365 Days of Masterpieces. Also, some applications are suitable for iPad, such as Auspicious Symbols in the Forbidden City and Twelve Beauties of Prince Yong. Different smart devices have different screen sizes, which will have different picture quality and display layouts. Therefore, when developing and designing digital cultural and creative products, the Palace Museum should take into account the differences of different terminal equipment, screen proportion, image quality and interaction design. In addition, according to the different use of mobile terminals, different user needs should be 
analyzed and targeted positioning and analysis should be made.

Based on the needs of users, the Cultural creation application of the Palace Museum combines digital media technology, digital mobile terminal devices and social media to further serve the needs of cultural information communication of museums and mass culture consumption. Application of the Palace Museum has two technical characteristics: interactive and immersive experience. First, the interaction design process includes the description of various user events and how the system responds to user requests [4]. Development of the imperial palace of Auspicious Symbols in the Palace Museum, The user can click on the design of various auspicious objects, open the display content such as interaction, convenient for the user to read the relevant cultural relics information, can magnify the details of the paintings, can browse different times with an auspicious symbol of cultural products, at the same time can be forwarded by a key to social media, to share the feeling of the auspicious culture symbol study. Secondly, immersive experience combines users' sensory experience with digital technology experience, maximizes visual, tactile and auditory sensory stimulation, and immerses the audience in the cultural and artistic space created by the APP. For example, the Night Revels of Han Xizai developed by the Palace Museum uses music, story narration and animation display to introduce the app's operation interface, which can be appreciated in-depth details through amplification. The texture and pattern in the painting are clear and visible. At the same time, it is also equipped with explanation narration, scholar Video Explanation and "real person" painting performance, giving people a sense of immersive experience. The digital cultural and creative products of the Palace Museum enrich the communication mode of the museum's art, combine the artistic content with digital technology, and let the audience interact with the "cultural relics in the collection". Compared with the traditional way of tourists explaining the cultural connotations of the museum through offline tour, the digital cultural and creative products of the Palace Museum spread the cultural connotations of the museum more rapidly through online promotion, analysis and social media sharing. At the same time, the emergence of digital cultural and creative products of the Palace Museum has better expanded the communication channels of cultural symbols of the Palace Museum, made up for the previous limitations of museums in time and space, and enhanced the social influence of the Palace Museum.

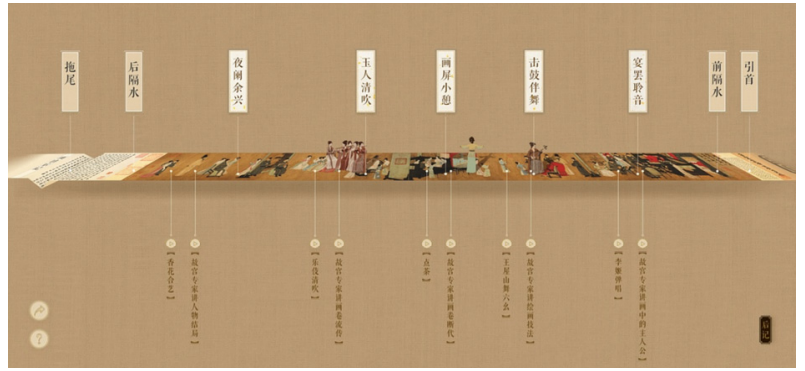

Figure 1 The frame of Night Revels of Han Xizai

\section{CASe Analysis of Twelve Beauties of PRINCE YoNG}

In May 2013, the Palace Museum released its first official iPad app, Twelve Beauties of Prince Yong. As to mobile digital media platform with certain experimental properties, the Palace Museum did not build its first product on the mobile terminal into a comprehensive tour guide tool, nor did it cooperate with the physical exhibition. Instead, they independently selected the collection for planning, adopted a small incision and indepth excavation method, and took the twelve fine brushwork ladies' paintings in the Qing Dynasty palace "Prince Yong's book Hall living in the deep screen" as the material. While interpreting the paintings, they introduce the same or similar information about the cultural relics in the collection as the furniture display in the background [5].

\subsection{The symbolic representation of Twelve Beauties of Prince Yong}

There are two types of symbol images in the APP Twelve Beauties of Prince Yong, namely functional symbol images and content symbol images. The APP of Twelve Beauties of Prince Yong aims to introduce and display relevant knowledge about the painting to the public, so the artistic connotation of the painting is the source of the overall content design, and functional symbols must serve and point to the content symbols. In the APP Twelve Beauties of Prince Yong, most of the functional symbols originate from Chinese calligraphy and painting styles. Main text navigation using ancient photocopy stylized font, vertical typesetting for layout design. The background is light beige in rice paper color, and the corner is decorated with seasonal flower symbols extracted from the painting. Although they are function symbols, but with aesthetic form of the original painting on the symbolic elements to keep highly consistent. The functional icons and decorative pairs of the interface must come from the symbol set contained in the theme collection, which ensures the stability and continuity of the user's aesthetic emotion in the process of content interaction. Since Twelve Beauties of Prince Yong rendered in the mainly content of image symbol, it is difficult to express symbol content, such as sound, movement. However, through the digital media technology, it can not only display the original image symbol content in the painting, but also express the information symbols such as voice and action, forming a complete space symbol code. At the same time, it is also conducive to the public to understand the cultural symbols with complex content such as Twelve Beauties of Prince Yong.

The Palace Museum has also expanded digital products featuring emojis and input methods based on the APP Twelve Beauties of Prince Yong. Among the tweets mainly used for marketing promotion, the Twelve Beauties of Prince Yong. emoji package mainly consists of two parts: visual symbols and text symbols. It is symbolized by re-symbolized images of content symbols 
from the original paintings, which is the practice of recreating symbols. The text symbols are usually network buzzwords or very colloquial content, while the visual symbols are adapted from exaggerated and funny expressions and actions of Twelve Beauties of Prince Yong. The combination of network buzzwords and visual symbols changes the original meaning of the visual symbols and expands the meaning decoding space of the original symbols, so the figures in the Painting have more cultural connotations. Emoticons are used in tweets to make them have corresponding meanings and interact with the public outside the APP, so as to promote the content encoded by topic symbols and connect with the society.

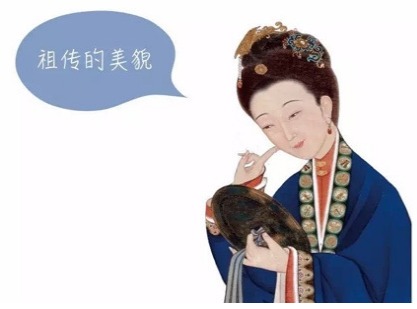

Figure 2 Emojis for Twelve Beauties of Prince Yong

Commodities not only have their own use value, but also have additional symbol value, and such symbol consumption often becomes the link between individuals and groups through the establishment of links. The common views, educational level and aesthetic orientation of groups are the essence of this link. In Twelve Beauties of Prince Yong, the Palace Museum constructed a huge symbol system through coding space and topics, and further reified it, so as to arouse the audience's aesthetic experience and emotional and cultural consumption demand.

\subsection{Theoretical analysis of digital technology in Twelve Beauties of Prince Yong}

First, interaction design. Twelve Beauties of Prince Yong uses three of the standard gestures for iPad interaction: clicking to enter the next level or activate modal Windows, dragging and dropping to move mock magnifiers, sliding to switch between navigation pages and scroll through text in the background. All of the above gestures are standard gestures familiar to iOS device users. Simple and direct operations can avoid increasing user learning costs and allow users to focus on the experience and content. The first-level interface displays the entire painting axis in tiled form to maximize the utilization of space. ICONS are placed in fixed positions with reduced content and less interference. Unlike other apps, fixed navigation bar is set on the top, which is conducive to reducing the visual segmentation of the whole picture. After entering the second level and the third level, it also returns to the upper level through a fixed position, reducing the number of jumps between different levels and making the interface logic of the whole App simple and clear. The background part of the text and text slide at different levels, the simple dynamic effect of the page is added, and the conversion effect between the levels are all using the simple effect, so as not to increase the user's interpretation of the content, just to add a simple interactive experience.

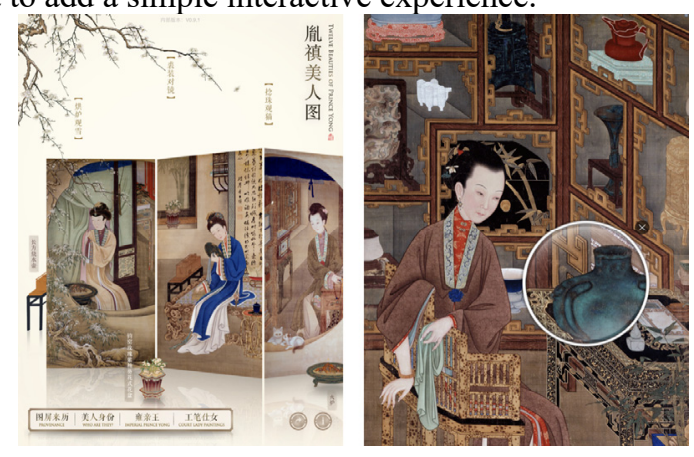

Figure 3 Interface display of Twelve Beauties of Prince Yong

Second, immersive experiences. Twelve Beauties of Prince Yong APP contains different research results of experts in the field of theoretical support. After in-depth mining and combing, and considering that the fragmented reading habits of the modern public are not suitable for long-term in-depth reading, the number of words is strictly controlled, part of the text content is visualized, so that the products and services created are more close to the user's habits. The structure of the product is in the form of database plus narrative context interface, which breaks the traditional linear narrative mode and gives users more space to explore freely. At the same time, in the early in the process of program development, in order to display the effect and content of the collection, the development team abandoned the smartphone terminal and focused on IOS tablet device. The size and display advantages of iPad device can maximize the visual impact and appeal of the color, pattern, line and other paintings. The addition of magnifying glass function in the firstlevel page of the product simulates the traditional way of appreciating artworks, so that users can see the details of cultural relics in a "close range". In the original painting of Twelve Beauties of Prince Yong, similar or identical pieces of paintings, furniture, accessories and patterns can be found in the Palace Museum. Information of these pieces can be further displayed by clicking on the products in the picture, forming a small "database" of the products for users to check. In addition, a simple animation effect is added to the first level interface. Butterflies flapping their wings, falling petals and bamboo leaves, Flickering candle light, etc, enter into each painting and the picture will slide slowly from top to bottom. These dynamic additions are intended to enhance the user's visual experience and the immersion of the program. In the appreciation of painting details, the analysis of composition is added. The composition is analyzed by using western painting composition methods and image processing of color block relationship, and the composition is analyzed by using Chinese painting text description, which enriches users' aesthetic and emotional experience.

\section{CONCLUSION}

In the era of digital media, the museum cultural and creative industry is facing many opportunities and 
challenges. The development of digital technology has brought more forms of products to the museum culture and innovation. Digital technology has strengthened the expression and special effects of cultural and creative products, and the public's cultural consumption choices have become more diversified. In the consumption environment, if digital cultural products want to succeed, they must improve the product quality, understand the consumer needs of the audience, do a good job of interactive and immersive experience analysis of digital products, and combine cultural symbols of cultural relics to output the symbolic meaning of digital products. The application of digital technology to the design of cultural and creative products is the development trend of the times. The Palace Museum should grasp the development direction of the market, create more excellent digital design products, and promote the development of my country's cultural industry.

\section{ACKNOWLEDGEMENT}

Project No.2017CDJSK05XK09 supported by the Fundamental Research Funds for the Central Universities

\section{REFERENCES}

1. Song Zhuyun.Brief Analysis of media Communication Characteristics of the Daily Palace Museum APP [J]. Popular Arts, 2016:180

2. Shi Siwen. Self-identity and Symbol Consumption: Observing code Information Construction of documentary programs -- A Case study of My Little Girl [J]. Film and Television,20

3. Wu Changwen. APP under the Wave of Mobile Internet and its Application in museums [J]. Fujian Wenbo,2012(2):76.

4. Zhao Huiwen, Zhang Jianjun. Network User Experience and Interaction Design [M]. Beijing: Higher Education Publishing,2012:16.

5. Zhuang Ying. Creating an app featured by the museum -- iPad app Planning of Twelve Beauties of Prince Yong in the Palace Museum. China Museum,2017(2):77 